\title{
Mobil FSO activities in Europe and fading mitigation approaches
}

\author{
Hennes HENNIGER ${ }^{1}$, Bernhard EPPLE ${ }^{1}$, Dr. Dirk GIGGENBACH \\ ${ }^{1}$ Institute of Communications and Navigation, German Aerospace Center (DLR), Oberpfaffenhofen, 82234 Wessling, \\ Germany \\ hennes.henniger@dlr.de, bernhard.epple@dlr.de, dirk.giggenbach@dlr.de
}

\begin{abstract}
The increasing emergence of data services for mobile applications requires high-speed communication technologies. To this end, free-space optical (FSO) communications technology has the potential to outperform radio frequency $(R F)$ systems. Within the last years, the German Aerospace Center (DLR) has performed several demonstrations of mobile FSO systems. A brief overview of these activities is given in this paper. Mobil FSO mainly suffers from relatively long link outages, produced by temporary obscured laser-beams, pointing- and trackingerrors or deep signal-fades caused by index of refraction turbulence effects. Error correction and retransmission techniques for fading mitigation are discussed in this paper. Comparison of FEC and protocol based error correction for mobile FSO transmission is presented.
\end{abstract}

\section{Keywords}

Free-space optics, mobile optical links, error correction, FEC, ARQ, TCP.

\section{Overview on mobile optical link experiments}

The subject of mobile free-space optical communications (MFSO) in space and inside the atmosphere is developed in response to the growing need of high-speed and tap-proof communications between and from satellites and deep-space probes, from unmanned aerial vehicles (UAVs), high altitude platforms (HAPs) and between aircraft and nomadic communications partners, both in military and civilian context.

While fixed optical communication links between buildings have been established long ago and today form a separate commercial product segment in LAN- and MANnetworks, the mobile application of this technology is aggravated by the extreme requirements on the pointing and tracking accuracy due to the small optical beam divergence. The later is necessary to fully exploit the benefits of optical links. Furthermore, long-haul optical links through the atmosphere suffer from strong fading caused by index-of-refraction turbulence and of course from link-blockage by clouds. Despite these limitations, mobile free-space optical communications is a subject of keen interest as no other communications technology even comes close in offering its transmission bandwidth while at the same time featuring smallest "antenna" (telescope) sizes and lowest power consumption. Furthermore, atmospheric optical free-space communications is not subject to frequency spectrum regulation and offers natural tap-proofness through its minimal signal spread.

Typical values for MFSO-terminals are signaldivergence angles between $1 / 1000$ to $1 / 100$ of a degree, some $100 \mathrm{~mW}$ to a few Watts of Tx-power, telescopediameters between few $\mathrm{cm}$ to $4 \mathrm{dm}$ and data rates from several hundred Mbit/s to several Gbit/s.

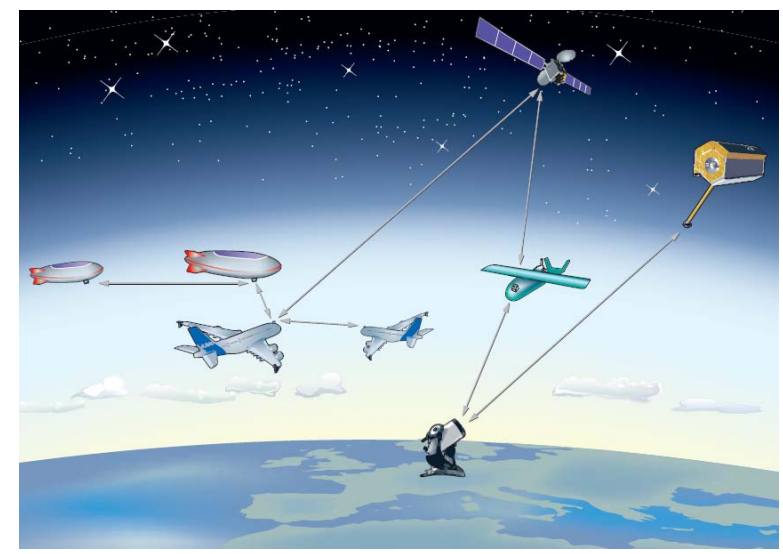

Fig. 1. Overview MFSO-Communications Scenarios.

ESA has launched the geostationary Satellite Artemis ("Advanced Relay and TEchnology MISsion") in 2001, which carries an optical communications terminal [1]. It has been used successfully for optical inter-satellite-link (OISL) tests with the earth-observation satellite SPOT-4 (which carries an according counter-terminal) as well as for GEO-ground links to ESA's optical ground station on Tenerife, Canary Islands.

Besides the generic space application of OISLs which are not susceptible to atmospheric effects - the satellite downlink from low earth orbit earth-observation satellites is a very promising future technology to overcome the downlink-bottleneck in earth-observation missions. To demonstrate the feasibility and robustness for this approach, DLR carried out the KIODO-experiment 
(Kirari Optical Downlink to Oberpfaffenhofen) together with the Japan Aerospace eXploration Agency [2]. The Japanese test satellite Kirari carries an optical communications terminal which was pointed towards DLR's optical ground station during eight overflights during June 2006, of which five trials were successful, while the other three suffered from cloud blockage. Uncoded bit error rates down to $2 \mathrm{E}-6$ could be measured using conventional intensity-modulation direct detection (IM/DD) transmission.

With the EU-FP6 project CAPANINA (Communications from Aerial Platforms providing high bandwidth communications for All) the development of a real long-range communications terminal was triggered in 2003. To test and prove the feasibility of optical inter-HAP communication as backbone links for a HAP communication network, the DLR built a prototype stratospheric optical terminal which was flown to the stratosphere at $22 \mathrm{~km}$ altitude with a remotely controlled balloon and delivered data at $1.25 \mathrm{Gbps}$ down to an optical ground station (OGS) [3]. Signal wavelength was $1550 \mathrm{~nm}$ with $100 \mathrm{~mW}$ transmit power, a transmit aperture of $5 \mathrm{~cm}$ diameter at the flight terminal and a $40 \mathrm{~cm}$ receive aperture at the OGS. This trial was executed at ESRANGE (European Sounding Rocket test range) near Kiruna, Sweden, in August 2005. The distance was up to $64 \mathrm{~km}$ and signal quality (in terms of BER) was nearly impeccable.

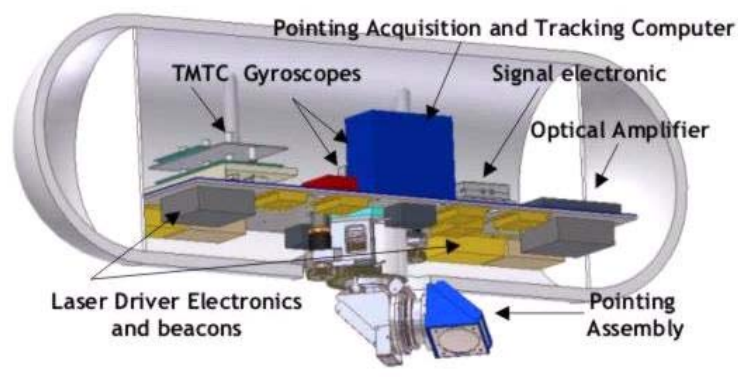

Fig. 2. Internal structure of the stratospheric optical terminal designed for the CAPANINA-trial

The experiment LOLA - a link between an aircraft and ESA's geostationary telecom test satellite ARTEMIS was performed by Astrium, Toulouse, with several successful trials in December 2006. The technology used is similar to the one of KIODO, as both systems are based on the same IM/DD transmission technology with $850 \mathrm{~nm}$ laser diode sources.

To demonstrate the feasibility of optical links from highly dynamic carriers, DLR carried out the MONDproject (Mobile Optical Near-ground Demonstrator) together with Carl-Zeiss Optronics, Oberkochen. The challenge here was to compensate the strong vibrations and fast attitude changes of a coasting bus with GPS-assisted inertial navigations sensors (INS) together with an eye-safe optical communications and tracking system at $1550 \mathrm{~nm}$ wavelength. High-definition video (HDTV) data at 1.5 Gbit/s was transmitted over a distance of $2 \mathrm{~km}$. Robust tracking and reacquisition even with link blockages was ensured by Kalman filtering tracking algorithms.

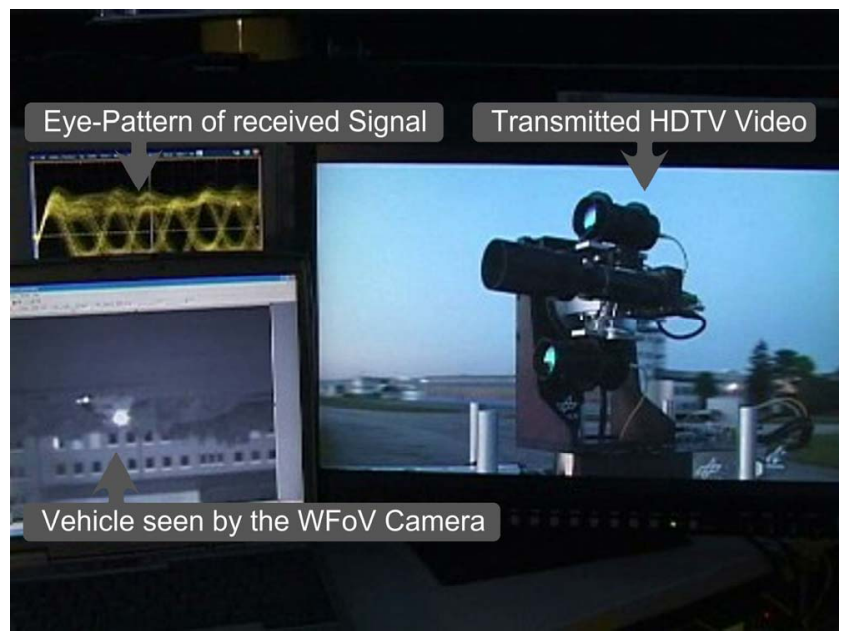

Fig. 3. Transmitted HDTV-Video from a moving van. The Van can be observed on the fixed terminal wide field of view (WFoV) camera. The transmitted video shows the mobile terminal optics. Also the current eye-pattern of the received signal is given [6].

The next huge step in MFSO development will be made with the launch of the German SAR satellite TerraSAR-X which carries a coherent optical high-speed communications terminal as secondary payload, build by Tesat-Spacecom, Germany, under DLR contract. This terminal transmits at $5.6 \mathrm{Gbit} / \mathrm{s}$ over up to $6000 \mathrm{~km}$ in space with $700 \mathrm{~mW}$ transmit power, using coherent BPSK homodyne reception. DLR will use this terminal to test the coherent space-ground link and investigate its susceptibility to atmospheric index-of-refraction turbulence (IRT).

\section{Brief channel description of optical fading channel}

One of the biggest challenges facing FSO deployment is its optical signal propagation in different atmospheric conditions. This effect will cause variable link degrading due to variable attenuation and fading. Atmospheric turbulence produces temporary pockets of air with slightly different indices of refraction. Therefore the laser beam phase-front varies randomly, producing intensity fluctuation. The duration of fades respectively the channel coherence time depends mainly on the relative orthogonal speed of the beam pathing through the pockets of air. The relative speed depends on wind speed or mobility of the scenario. For stationary links time constants in the order of milliseconds to tens of milliseconds can be observed, while fluctuations are faster in highly mobile scenarios, like aeronautical links. One further key challenge with FSO systems is maintaining transceiver alignment. Using highly directional and narrow beams of light, variable misspointing of the transmit-beam, and tracking-errors of the receiver will generate additional signal fading. Further 
link blockings by line-of-sight obscuration or hand-over procedures from one partner to another can produce extremely long link outages in the time frame of seconds. Generally the optical fading channel can be said to be a non frequency selective very slow fading channel. Considering a short link outage produced by atmospheric turbulence of $1 \mathrm{~ms}$ and a data-rate of only $100 \mathrm{Mbit} / \mathrm{s}$ an error burst of 100.000 bits occurs. With a packet-based transmission like Ethernet, which can use a maximum packet-length of approximately 1500 byte, only about 10 packets will be affected by this fade. If the packet-based transmission implements CRC (cyclic redundancy check), like Ethernet does, these 10 packets will be discarded and not delivered to higher layer applications. In this case the channel can be modeled as a packet-erasure channel (PEC).

\section{Reliable content delivery}

\subsection{Performance of transport-layer FEC}

Large difference of channel coherence time and bit duration in the FSO channels causes problems if working with physical-layer coding to mitigate link blockings and fades. Interleaving over some milliseconds to seconds will be needed in order to avoid erasure of whole codewords during fades. For high data-rates these interleavers are technically not feasible. Packet-layer coding for example on transport layer makes it possible to generate codewords longer than fades. Depending on the data-rate code-symbol length will be in the same order as fade-duration.

In transport-layer coding the sender side consists of a device generating packets based on objects (e.g. large files) to be delivered within the session. It further generates encoding symbols based on the object to be delivered using FEC codes and sends them in packets to the receiver. Receiver simply waits for enough packets to arrive in order to reliably reconstruct the object. An example for transport layer coding is described in RFC3450: ALC (asynchronous layered coding protocol) which is a reliable content delivery protocol based on IP multicast. ALC implements FEC on transport layer to ensure correct object transmission.

Let $\mathrm{n}$ be the number of symbols in a codeword, $\mathrm{k}$ the number of data symbols in a word, then $n-k$ is the number of parity symbols. $\mathrm{R}$ is the redundancy. For error-free transmission a rule of thump exists which says that $\mathrm{R}$ should be approximately $10 \%$ greater than the expected probability of packet-loss [4]. The additional transmitted parity packets compared to the number of data packets define the throughput.

Throughput values can be calculated using equation (1). Plots comparing FEC and ARQ throughput are given in the following sections. It can be seen that the throughput of ARQ is always higher but this advantage can settle down if the additional effort for the needed return channel is counted. Depending on the code a nearly zero residual error probability can be ensured if the above given design rules are applied [4], [5].

$$
\begin{aligned}
& R=\frac{n-k}{n} \\
& R=p+0.1 \\
& \eta_{t h}=1-\frac{\text { parity }}{\text { data }}=1-\frac{n-k}{k} \\
& \eta_{t h}=2-\frac{1}{1-p-0.1}
\end{aligned}
$$

In the following a measurement example is presented. In a mobile optical link with $100 \mathrm{Mbit} / \mathrm{s}$ Ethernet over a distance of $1.5 \mathrm{~km}$ the following code was used: Number of data packets: 100.000 . Number of parity packets: 50.000 . Therefore the redundancy is $33 \%$. In a transmission 39248 packets were lost. That means packet-loss rate $\mathrm{p}$ is $26 \%$. So here the code has $7 \%$ more redundancy than the packetloss is and it was able to recover all data-packets without any residual error.

\subsection{Performance of standard ARQ}

At first the standard internet ARQ protocol TCP is investigated.

If round trip time RTT is constant, packet-loss is random at constant probability $\mathrm{p}$, and TCP operates without timeouts, the upper bound of TCP-performance is given in [7] as:

$$
D R_{T C P, p}<\frac{M S S}{R T T} \cdot c \cdot p^{-0.5}
$$

$\mathrm{c}$ is a constant describing the influence of the acknowledge mechanism. For delayed acknowledge algorithm (DA) it can be set to 1 . MSS is the maximum segment size (packetsize). The equation (2) is only applicable if the receiver window is large enough. Receiver window size $\mathrm{N}$ limits the TCP throughput to:

$$
D R_{T C P, w}<\frac{N}{R T T}
$$

When the average loss-rate $p$ is large, equation (2) becomes inaccurate. In the case of high loss rates, the loss of multiple packets per RTT becomes likely. In this case TCP will lose its self-clock and will start a slow-start recover. For $p>0.1$ the timeout significantly reduces the throughput and equation (2) overestimates the possible throughput. Nevertheless we will use (2) to verify optimistically TCP performance in an analytical way.

Figure 4 shows TCP is only applicable effectively for low loss-rates. 


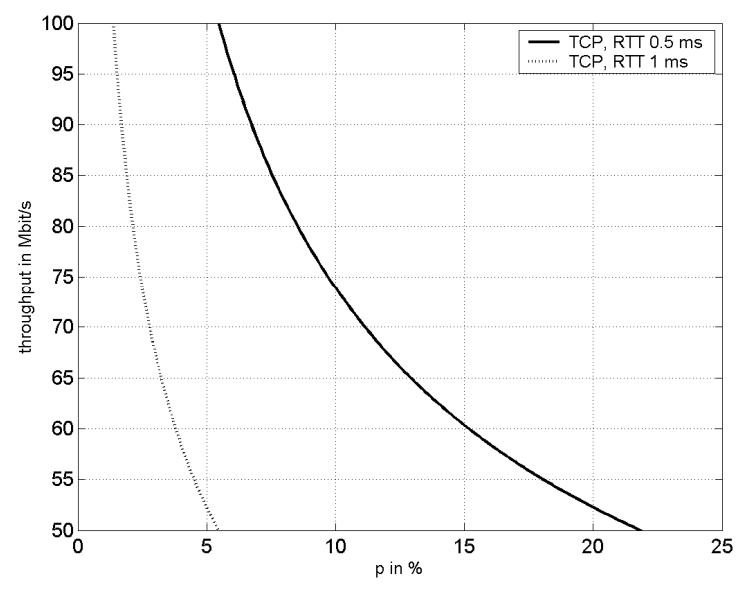

Fig. 4. TCP throughput $[\mathrm{Mbit} / \mathrm{s}](\mathrm{TCP} 1 \mathrm{with} 0.5 \mathrm{~ms} \mathrm{RTT}$ and TCP2 with $1 \mathrm{~ms}$ ) for different probabilities of packetloss $\mathrm{p}$ [\%]; according to equation (2); MSS $=1468$ byte. Eq. 3 gives for $\mathrm{N}=64$ Kbyte and TCP2 a max. data-rate of about $0.5 \mathrm{Gbit} / \mathrm{s}$

In addition it has to be considered that TCP needs a return channel. For using delayed acknowledge strategy the needed channel capacity for the return channel $\mathrm{DR}_{\mathrm{TCP}, \mathrm{r}}$ depends on the (maximum) forward channel data-rate $\mathrm{DR}_{\mathrm{TCP}, \mathrm{f}}$ :

$$
D R_{T C P, r}=\frac{N_{A C K}}{2 \cdot M S S} \cdot D R_{T C P, f}
$$

This equation only considers acknowledge packets.

The length of a TCP acknowledge packet is by default 40 bytes. So the needed return channel capacity is approximately $1.3 \%$ of forward channel data-rate, for Ethernet's MSS (segment size is assumed to be constant in this case).

As it can be seen in figure $4 \mathrm{TCP}$ has problems with a large amount of packet-loss and high delay times. Therefore the performance of the more suitable SelectiveRepeat ARQ is investigated in the following.

With Selective-Repeat ARQ data blocks are transmitted continuously. The receiver asks for retransmission until data is transmitted completely. Selective-repeat ARQ is the most efficient ARQ however complexity and memory requirement is ambitious.

At a time a special amount of data $M$ is transmitted plus additionally the data which was asked for repeat. An optimal return-channel without ACK/NAK losses and a constant probability of loss $\mathrm{p}$ is assumed. Throughput is reduced by additional repeat request data. With a probability of packet-loss $\mathrm{p}$, data from the last transmission event is requested to retransmit and with a probability of $\mathrm{p}^{2}$ from the event before last, and so on. So the overhead is given by:

$$
\begin{aligned}
& M\left(p+p^{2}+\ldots p^{\infty}\right) \\
& =M \sum_{i=1}^{\infty} p^{i} \rightarrow \frac{1}{1-p}-1
\end{aligned}
$$

Therefore the channel data-rate DR is reduced in the case of Selective-Repeat ARQ to $\mathrm{DR}_{\mathrm{ARQ}}$ :

$$
\begin{aligned}
& D R_{A R Q}=D R\left[1-\left(\sum_{i=1}^{N_{\max }} p^{i}\right)\right] \\
& \underset{N_{\max }=\infty}{\longrightarrow} D R\left[1-\left(\frac{1}{1-p}-1\right)\right]
\end{aligned}
$$

In a protocol implementation one will reduce the number of retransmission events to a certain number of $\mathrm{N}_{\max }$. This will enhance the throughput a little bit but with the disadvantage of having a certain residual error probability. Figure 5 below gives the throughput-efficiency calculated by

$$
\eta_{t h}=\frac{D R_{A R Q}}{D R}=2-\frac{1}{1-p}
$$

in case of infinite retransmissions and in case of only one retransmission $\left(\mathrm{N}_{\max }=1\right)$; according to equations (6) and (7).

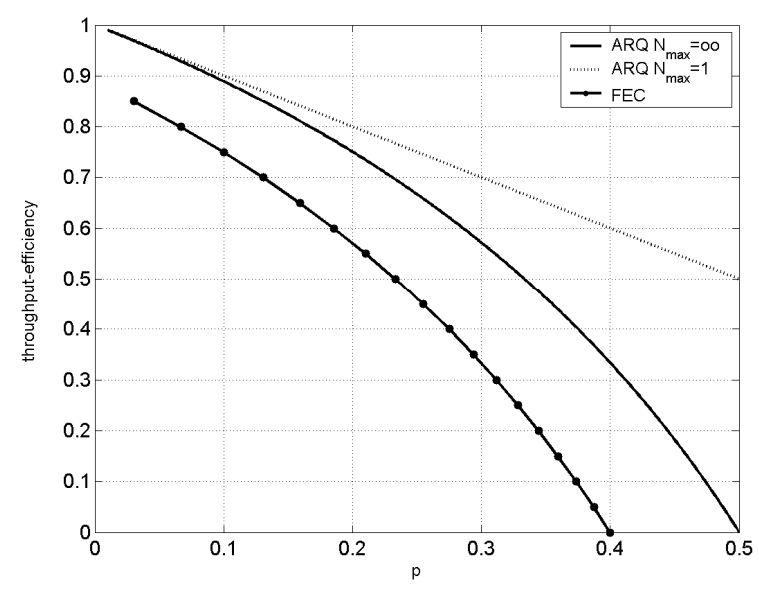

Fig. 5. Throughput-efficiency of Selective-repeat ARQ vs. packet-loss for infinite retransmission actions and for the case that a packet is requested only one time. For comparison also FEC throughput efficiency according to equation (1) is presented.

The residual error probability is depending on $\mathrm{N}_{\max }$ as well as on the packet-loss probability $\mathrm{p}$. As shown above in figure 5, ARQ with $\mathrm{N}_{\max }=\infty$ is not applicable for $\mathrm{p}>0.5$. The next figure gives an impression on what residual error one has to expect for different $\mathrm{N}_{\max }$ 


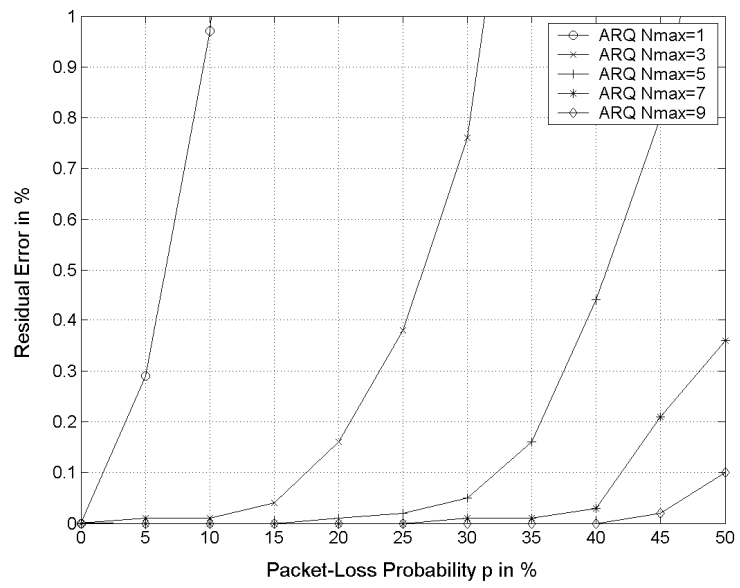

Fig. 6. Residual Error for different $\mathrm{N}_{\max }$ values in relation to $\mathrm{p}$. Values are taken from OmNet++-Simulations

Figure 6 shows the results of simulations with the OmNet++ simulation framework [8]. For simulation, 10000 packets with a size of 8000 bit where transmitted. In the simulation, the transmit requests where sent over an error free backchannel. The plot shows that the more iterations are allowed, the less the residual error is; so it might be desirable to allow as many iterations as possible. Figure 7 below shows that an ARQ protocol with $\mathrm{N}_{\max }=1$ has the highest throughput, but it bears also a large residual error (see Figure 6) which can not be accepted in most applications.

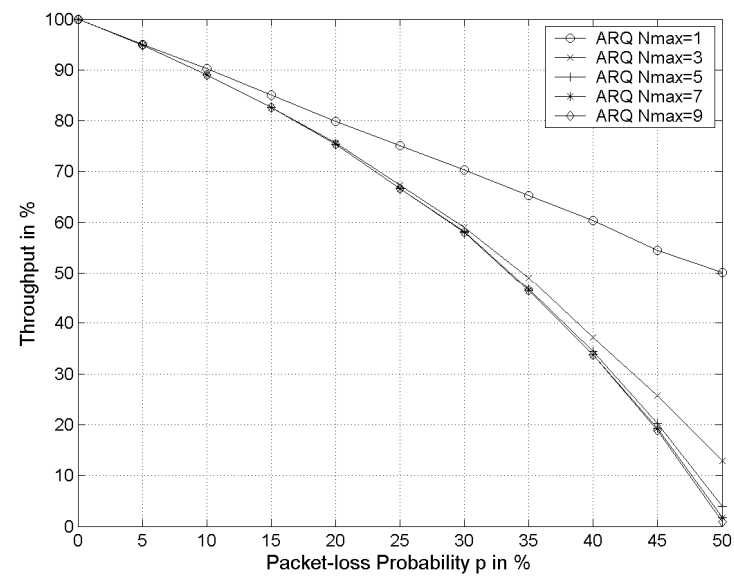

Fig. 7. Simulated throughput-efficiency of Selective-repeat ARQ compared against packet-loss. Simulation parameters are the same as for Figure 6

For higher $\mathrm{N}_{\max }$ values ( $>3$ ) the throughput stays in the same range. For a low residual error one should implement as many retransmissions as possible. The reduced throughput does not justify why one should not implement an ARQ with $\mathrm{N}_{\max }=\infty$. The limiting factor on the number of allowed retransmissions is the maximum allowed transmission delay for the application data, since retransmission iterations are generally timeout-triggered. So an increased $\mathrm{N}_{\max }$ results in an increased maximum transmission delay which is the product of $\mathrm{N}_{\max }$ and the timeout duration. Since all not confirmed or timed out packets have to be stored at the sender, a high $\mathrm{N}_{\max }$ might also lead to buffering problems.

\subsection{Outlook on protocol requirements}

For securing a channel against data-loss the bit error probability $(B E P)$ of the channel is an important measure. Depending on the current $B E P$, packet-loss $p$ can be calculated as:

$$
p=1-(1-B E P)^{P L}
$$

Where $P L$ is the packet length. This can be developed to

$$
P L=\frac{\log (1-p)}{\log (1-B E P)}
$$

When designing a communication protocol (9) can be used to determine the maximum allowed packet length by using the expected $B E P$ and the desired packet-loss probability as input values. This formula is only valid for a constant BEP. As mentioned before, the BEP of the optical channel is changing over time, but it can be assumed as being constant over several thousands of bits. Formula (9) can be used in adaptive systems to recalculate the packet length, if the channel state is known.

The selection of error protection scheme depends on the channel type. If the communication is done via a unidirectional link FEC is the only choice for securing the channel. Unidirectional communication has the disadvantage of not being able to sense the link quality on the protocol level, since no channel information can be exchanged between sender and receiver; therefore FEC parameters have to be set in advance to fit the worst packet loss expected. By this fixture the throughput will be fixed and in cases where the channel has better quality than expected, available bandwidth will be wasted. Measurements with operational systems show that $B E R$ varies between $10^{-2}$ and $\sim 10^{-9}$ or even better. Another disadvantage of non-adaptive FEC is that in cases where the channel quality is worse than expected, the parameters can not be adjusted and data will be lost. To be able to work efficient, protocols should be able to sense the current link quality and adapt its parameters according to it. This implies the need of a backchannel for exchanging channel information.

On bidirectional channels ARQ based protocols can be used. This protocol class is able to sense the link quality and therefore can adjust its parameters to achieve a higher throughput. The easiest way for sensing the link quality is to calculate $p$ by keeping track of the number of sent packets and the number of retransmission requests. For this, formula (8) can be rewritten to:

$$
B E P=1-\sqrt[P L]{1-p}
$$

Since the optical channel does not have a constant $B E P$ this calculation is only valid for a short duration. So it should be implemented using a sliding window technique. 
The disadvantage of ARQ based protocols is that you have either to accept a residual packet-loss or to deal with buffering problems and long transmission delays due to a high number of retransmission requests on bad channels.

The FEC and the ARQ approach have both advantages and disadvantages, so it is good idea to combine them to a hybrid scheme. For combining the two schemes, the packets are coded for transmission over the channel in its currently sensed state. The additional information bits needed for worse channels conditions are stored at the sender. In case of a retransmission request, additional information bits are sent instead of the original packet. This step can be repeated until the receiver is able to reconstruct the sent data.

Coding systems are generally designed to fix bit errors in the transmitted data. To be suitable for the optical channel, these systems have to be adapted to the possibility of packet-loss. This can be done by implementing two types of retransmission requests; one for bit errors in the packet and one for a complete packet-loss. In case of a complete packet-loss the sender has to retransmit the last sent packet, in case of bit errors it has to send the next redundancy packet.

The sensed channel information is also important for use in FSO network routing protocols. Since FSO networks have a high change dynamic where the network topology is not fixed and can change rapidly, the routing protocols need frequent updates on link states to avoid sending data over broken links.

\subsection{Proposal on error protection design}

As it comes out packet-layer FEC is suitable for low and high loss rates if the redundancy is high enough. Designing the FEC for improperly cases of very high losses the overall throughput decreases. A hybrid system combining FEC and ARQ is appropriate: FEC will enhance packet-loss ratio and in the unlikely case that FEC can not deal with a too high amount of losses ARQ can be used to ensure a reliable data transmission. A block-diagram of such a hybrid system is given in the next figure.

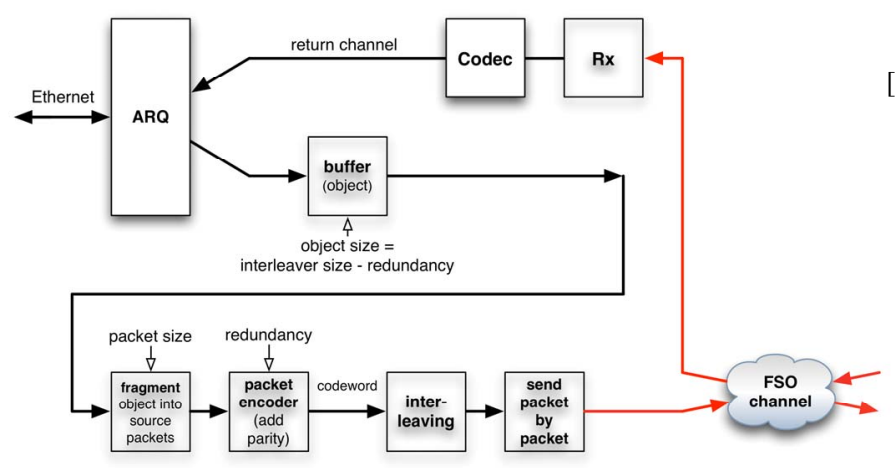

Fig. 8. System approach for effective error protection on a FSO fading channel: Hybrid ARQ and packet-FEC system

\section{Conclusion}

The great number of activities in the field of mobile free-space optics shows the increasing importance of high data -rate links provided by optics. Successful trials let the community believe that FSO could be a main component in future mobile network structures.

Error protection schemes can help to overcome weaknesses of optics. Packet-layer forward error correction shows its strength in a slow atmospheric fading channel with a great number of packet-losses. Anyway even the best code will fail if fades getting extraordinary long, e.g. produced by long link blockings or temporary terminal alignment problems. These events are very infrequent therefore ARQ can deal with this perfectly. Hence a hybrid system with inner packet-layer coding and outer ARQ error protection is favorably to guarantee a good quality of service.

\section{References}

[1] T. Tolker-Nielsen, G. Oppenhaeuser: "In Orbit test results of an Operational Optical Intersatellite Link between ARTEMIS and SPOT4, SILEX", Proceedings of the SPIE, Vol. 4635, 2002

[2] N. Perlot, M. Knapek, D. Giggenbach, J. Horwath, M. Brechtelsbauer, Y. Takayama, and T. Jono: "Results of the Optical Downlink Experiment KIODO from OICETS Satellite to Optical Ground Station Oberpfaffenhofen (OGS-OP)." Conference on Laser Communication and Propagation, Proc. of SPIE, 6457A, 2007.

[3] J. Horwath, M. Knapek, B. Epple, M. Brechtelsbauer, B. Wilkerson: "Broadband Backhaul Communication for Stratospheric Platforms: The Stratospheric Optical Payload Experiment", Proceedings of the SPIE, Vol. 6304, 2006

[4] H. Henniger, and A. Gonzalez: "Transmission Scheme and Error Protection for Simplex Long-Distance Atmospheric FSO Systems." Special issue of the Mediterranean Journal of Electronics and Communications on Hybrid RF and Optical Wireless Communications, 2006.

[5] H. Henniger: „Packet-Layer Forward Error Correction Coding for Fading Mitigation." Proc. SPIE, Vol. 6304, 630419, Free-Space Laser Communications VI, 2006.

[6] H. Henniger, and B. Epple:,,Free-space optical transmission improves land-mobile communications." SPIE Newsroom, 2006.

[7] M. Mathis, J. Semke, j. Mahdavi: "The Macroscopic behavior of the TCP congestion avoidance algorithm." Computer Communication Review, ACM SIGCOMM, Vol. 27, No 3, July 1997.

[8] A. Varga, "The OmNet++ Discrete Event Simulation System" In Proceedings of the European Simulation Multiconference (ESM'2001), June 2001. 